\title{
Application of In Vitro Release Methods to Assure Product Performanee of Semisolid Dosage Forms Before and After Certain Post-Approval Changes
}

\section{Intraduction}

In May 1997, FDA released a guidance entitled Scale-up and Post Approval Changes: Chemistry, Manufacturing and Controls, In Vitro Release Testing and In Vivo Bioequivalence Documentation for Nonsterile Semisolid Dosage Forms (SUPAC-SS) (1). The guidance focuses on nonsterile semisolid dosage forms such as creams, gels, lotions, and ointments. The document describes allowable changes in four separate categories, as follows: 1) components and composition; 2) manufacturing equipment and process; 3) scale (batch size); and 4) site of manufacture. Changes are categorized as Level 1, Level 2 and Level 3, depending on the degree of change and the type of tests needed to document comparability in identity, strength, quality, purity, and potency of the drug product before and after the change. Level 1 changes are those that are unlikely to bave any detectable impact on formulation quality and performance of the product. This degree of change does not require any additional testing for product approval beyond assurance that application and compendial specifications are met. Level 2 changes are those that could have a significant impact on formulation quality and performance of the product. For Level 2 changes, the guidance recommends in vitro release (IVR) testing in addition to assuring that application and compendial specifications are met. Level 3 changes are those that are likely to have a significant impact on formulation quality and performance of the product. This degree of change requires in vitro release test for a site change or in vivo bioequivalence testing for changes in component and composition, in addition to assurance that application and compendial specifications are met.

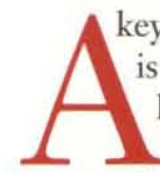

key aspect of the SUPAC-SS document is the recommendation that in vitro release testing be used under certain circumstances. The scientific principles and value of IVR have been debated over the last decade at several national and international meetings. At a recent scientific workshop entitled Assessment of Value and Applications of In Vitro Testing of Topical Dermatological Drug Products, held September 1997 in Arlington (2), IVR was concluded to be a property of the finished dosage form and could be applied to assure similarity in performance before and after certain changes. These conclusions were in agreement with those reached earlier in a prior workshop entitled ScaleUp of Liquid and Semisolid Disperse Systems, held May 1993 in Arlington, VA (3). IVR can be regarded as a 'final quality control' test that can signal possible inequivalence in performance, although it should not be used to document bioequivalence. In the guidance, this signal is suggested to occur when the the ratio of the median release rate for the post-change (test) product over the median release rate for the pre-change (reference) product is outside the $90 \%$ confidence interval limits of $75 \%$ to $133 \%$. Several papers (4-10), including a recent article by Zatz and Segers (10), have appeared in the literature describing the methodology and technique for measuring IVR from semisolid dosage forms. In this article, a general description of the methodology and a detail analysis of comparison of IVR rate is provided.

\section{The Role of In Vitro Release Testing}

The key parameter for any drug product is its efficacy as demonstrated in controlled clinical trials. The time and expense associated with such trials make them unsuitable as routine quality control methods. Therefore, in vitro surrogate tests are often used to assure that product quality Continued page 7
Vinod P. Shah* Jerome Elkins

Donald Schuirman Francis Pelsor

Surendra Shrivastava Wilson DeGamp

Paul Schwartz and Roger Williams

Office of Pharmaceutical Science Center for Drug Evaluation and Research,

Food and Drug Administration Rockville, Maryland

*To whom correspondence should be addressed.

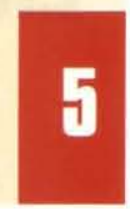




\section{Applieation of In Vitro Release Methods....cont.}

and performance are maintained over time and in the presence of change. A variety of physical and chemical tests commonly performed on semisolid products and their components (e.g., solubility, particle size and crystalline form of the active component, viscosity, and homogeneity of the product) have historically provided reasonable evidence of consistent performance. More recently, in vitro release testing has shown promise as a means to comprehensively assure consistent delivery of the active component(s) from semisolid products.

An in vitro release rate can reflect the combined effect of several physical and chemical parameters, including solubility and particle size of the active ingredient and rheological properties of the dosage form. In most cas$\mathrm{es}$, in vitro release rate is a useful test to assess product sameness between prechange and postchange semisolid products. However, there may be instances where it is not suitable for this purpose. In such cases, other physical and chemical tests with appropriate metrics and statistical approaches should be developed to document "product sameness."

\section{In Vitro Release Test}

In vitro release is one of several standard methods which can be used to characterize performance characteristics of a finished topical dosage form, i.e., semisolids such as creams, gels, and ointments. Important changes in the characteristics of a drug product formula or the thermodynamic properties of the drug(s) it contains should show up as a difference in drug release. Release is theoretically proportional to the square root of time $(\sqrt{t})$ when the formulation in question is in con- trol of the release process because the release is from a receding boundary.

In vitro release method for topical dosage form is based on an open chamber diffusion cell system such as a Franz cell system, fitted usually with a synthetic membrane. The test product is placed on the upper side of the membrane in the open donor chamber of the diffusion cell and a sampling fluid is placed on the other side of the membrane in a receptor cell. Diffusion of drug from the topical product to and across the membrane is monitored by assay of sequentially collected samples of the receptor fluid. The in vitro release methodology should be appropriately validated. Sample collection can be automated.

Aliquots removed from the receptor phase can be analyzed for drug content by high pressure liquid chromatography (HPLC) or other analytical methodology. A plot of the amount of drug released per unit area $(\mathrm{mcg} / \mathrm{cm} 2)$ against the square root of time yields a straight line, the slope of which represents the release rate. This release rate measure is formulation-specific and can be used to monitor product quality. The release rate of the biobatch or currently manufactured batch should be compared with the release rate of the product prepared af- ter a change as defined in this guidance.

One possible in vitro release study design is summarized below.

\section{Diffusion Cell System:}

- A diffusion cell system with a standard open cap ground glass surface with $15 \mathrm{~mm}$ diameter orifice and total diameter of $25 \mathrm{~mm}$.

\section{Synthetic Membrane:}

- Appropriate inert, porous and commercially available synthetic membranes such as polysulfone, cellulose acetate/ nitrate mixed ester, or polytetrafluoroethylene $70 \mu \mathrm{m}$ membrane of appropriate size to fit the diffusion cell diameter (e.g., $25 \mathrm{~mm}$ in above case).

Continued page 8 


\section{Application of In Vitro Release Methods.... cont.}

\section{Receptor Medium:}

- Appropriate receptor medium such as aqueous buffer for water soluble drugs or a hydro- alcoholic medium for sparingly water soluble drugs or another medium with proper justification.

\section{Number of Samples:}

- Multiple replicates (six samples are recommended) to determine the release rate (profile) of the topical dermatological product.

\section{Sample Applications:}

- About $300 \mathrm{mg}$ of the semisolid preparation is placed uniformly on the membrane and kept occluded to prevent solvent evaporation and compositional changes. This corresponds to an infinite dose condition.

\section{Sampling Time:}

- Multiple sampling times (at least 5 times) over an appropriate time period to generate an adequate release profile and to determine the drug release rate (a 6-hour study period with not less than five samples, i.e., at 30 minutes, 1, 2, 4 and 6 hours) are suggested. The sampling times may have to be varied depending on the formulation. An aliquot of the receptor phase is removed at each sampling interval and replaced with fresh aliquot, so that the lower surface of the membrane remains in contact with the receptor phase over the experimental time period.

\section{Sample Analysis:}

- Appropriate validated specific and sensitive analytical procedure should be used to analyze the samples and to determine the drug concentration and the amount of drug released.
In Vitro Release Rate:

- A plot of the amount of drug released per unit membrane area $\left(\mathrm{mcg} / \mathrm{cm}^{2}\right)$ versus square root of time should yield a straight line. The slope of the line (regression) represents the release rate of the product. An X intercept typically corresponding to a small fraction of an hour is a normal characteristic of such plots.

\section{Design of the Rate (Profile) Comparison Study:}

- The typical in vitro release testing apparatus has six cells. For each run of the apparatus, the two products being compared should be assigned to the six cells as follows:

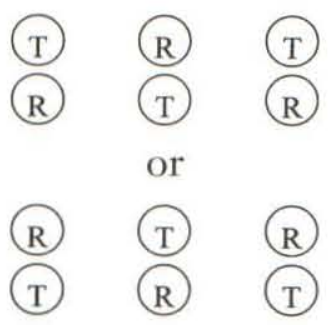

where $\mathrm{T}$ represents the Postchange Lot (Test product) and $\mathrm{R}$ represents the Prechange Lot (Reference product). This approach of including both products in each run of the in vitro apparatus will help ensure an unbiased comparison in the event of a systematic difference between runs.

- The choice of the assignment of products to cells (i.e., whether the prechange lot or the postchange lot is assigned to the "upper left corner cell" of the apparatus) may either be made systematically (i.e., alternate the pattern for each successive run) or randomly (i.e., flip a coin or use some other random mechanism).

- For the case of a nonstandard apparatus, with other than six cells, the principle of including both the prechange lot and the postchange lot in the same run should still be used. If the apparatus has only a single cell, the runs on the prechange and postchange lots should be intermixed, rather than obtaining all observations on one product followed by all observations on the other product.

\section{Details of the In Vitro Release Comparison Test}

-The in vitro release comparison should be carried out as a two-stage test.

At the first stage, two runs of the (six cells) in vitro apparatus should be carried out, yielding six slopes (estimated in vitro release rates) for the prechange lot (R) and six slopes for the postchange lot (T). A 90\% confidence interval (to be described below) for the ratio of the median in vitro release rate (in the population) for the postchange lot over the median in vitro release rate (in the population) for the prechange lot should be computed and expressed in percentage terms. If, at the first stage, this $90 \%$ confidence interval falls within the limits of $75 \%$ to $133.33 \%$, no further in vitro testing is necessary.

If the test is not passed at the first stage, 4 additional runs of the (six cells) in vitro apparatus should be carried out, yielding 12 additional slopes for each product, or 18 in all (including the first-stage results). The $90 \%$ confidence interval (described on page 10) should be computed using all 18 slopes for each product, including the first-stage results. At the second stage, this $90 \%$ confidence interval should fall within the limits of $75 \%$ to $133.33 \%$. continued on page 10 


\section{Application of In Vitro Release Methods....cont.}

\author{
Computation of Confidence \\ Interval - an Example: \\ - Because outliers are expected \\ to occur on occasion with this test- \\ ing (for example, due to an air bub- \\ ble between the product sample \\ and the membrane), a nonpara- \\ metric method is proposed, whose \\ performance tends to be resistant \\ to the presence of outliers. The \\ computations are illustrated in the \\ following example: \\ Suppose that the slope data \\ obtained at the first stage are as \\ follows:
}

\begin{tabular}{|c|c|}
\hline Postchange & Prechange \\
\hline $\operatorname{Lot}(\mathrm{T})$ & Lot $(\mathrm{R})$ \\
\hline 1.3390 & 1.1331 \\
\hline 1.3496 & 1.1842 \\
\hline 1.4946 & 1.0824 \\
\hline 1.4668 & 1.3049 \\
\hline 1.1911 & 1.0410 \\
\hline 1.2210 & 1.2419 \\
\hline
\end{tabular}

The first step in the computation of the confidence interval is to form the $36(=6 \times 6)$ individual $T / \mathrm{R}$ ratios. This is illustrated in the following table, where the prechange lot slopes (R) are listed across the top of the table, the postchange lot slopes $(\mathrm{T})$ are listed down the left margin of the table, and the individual T/R ratios are the entries in the body of the table:
1.2863, 1.2945, 1.2964, 1.3190, $1.3551,1.3808,1.4090,1.4357$.

In the third step, the $\boldsymbol{e i g h t h}$ and twenty-ninth ordered individual ratios are the lower and upper limits, respectively, of the $90 \%$ confidence interval for the ratio of the median in vitro release rate (slope) for $\mathrm{T}$ over the median in vitro release rate for $\mathrm{R}$. In the example, this confidence interval is 1.0343 to 1.2863 , or in percentage terms,

$$
103.43 \% \text { to } 128.63 \% \text {. }
$$

Because this confidence interval falls within the limits of $75 \%$ to $133.33 \%$, the product passes at the first stage.

If the product had not passed at the first stage, an additional 4 runs would have been carried out, yielding 12 additional slopes per lot, for a total of 18 slopes per lot altogether (including the firststage slopes).

All $324(=18 \times 18)$ individual $\mathrm{T} / \mathrm{R}$ ratios would be obtained, and these would be ranked from lowest to highest. It should be evident that even the computations at the first stage would be tedious to do by hand, and doing the computations at the second stage by hand is infeasible. A computer

\begin{tabular}{|l|llllll|}
\hline \multicolumn{1}{c}{} & 1.1331 & 1.1842 & 1.0824 & 1.3049 & 1.0410 & 1.2419 \\
\cline { 2 - 7 } 1.3390 & 1.1817 & 1.1307 & 1.2371 & 1.0261 & 1.2863 & 1.0782 \\
1.3496 & 1.1911 & 1.1397 & 1.2469 & 1.0343 & 1.2964 & 1.0867 \\
1.4946 & 1.3190 & 1.2621 & 1.3808 & 1.1454 & 1.4357 & 1.2035 \\
1.4668 & 1.2945 & 1.2386 & 1.3551 & 1.1241 & 1.4090 & 1.1811 \\
1.1911 & 1.0512 & 1.0058 & 1.1004 & 0.9128 & 1.1442 & 0.9591 \\
1.2210 & 1.0776 & 1.0311 & 1.1280 & 0.9357 & 1.1729 & 0.9832 \\
\hline
\end{tabular}
should be used.

At the second stage, the 110th and the 215th ordered individual ratios are the lower and upper limits, respectively, of the $90 \%$ con-

The second step in the com10 putation of the confidence interval is to order these 36 individual T/R ratios from lowest to highest: $0.9128,0.9357,0.9591,0.9832$, $1.0058,1.0261,1.0311,1.0343, \ldots$ fidence interval for the ratio of the median in vitro release rate (slope) for $\mathrm{T}$ over the median in vitro release rate for $\mathrm{R}$. If this confidence interval falls within the limits of $75 \%$ to $133.33 \%$, the product passes the test at the second stage.

\section{Further Remarks on the In Vitro Release Comparison Test}

- The statistical test described above is based on a standard confidence interval procedure related to the Wilcoxon Rank Sum/Mann-Whitney rank test, applied to the $\log$ slopes $(11,12)$. However, as was seen in the example, it is not necessary to actually compute logs in order to carry out the test.

- The example illustrates the case of full data, i.e., where there are 6 slopes per lot at the first stage and, if the second stage is necessary, 18 slopes per lot at the second stage. If slopes are missing, the computations will need to be modified. For example, if a single slope were missing from one of the lots (it does not matter if it is the prechange lot or the postchange lot) at the first stage, there would only be $30(=5 \times 6)$ individual $\mathrm{T} / \mathrm{R}$ ratios, and the limits of the $90 \%$ confidence interval would no longer be the eighth and twenty-ninth ordered individual $T / R$ ratio, but rather would be the sixth and twenty-fifth ordered individual $T / R$ ratio. If data are missing at either stage of the test, the correct computation should be determined by reference to a statistical text.

- The statistical procedure as described above does not take the block structure of the test (i.e., the fact that data are obtained in runs of six slopes at a time, rather than all at once) into account. This is justified by the following:

1. In vitro release data available to the Center at this time show no evidence of an important run-to-run effect.

2 . The proposed experimen- 
tal design, in which both products are included in each run, will help to ensure unbiased results if a run-to-run effect should occur.

\section{Conclusion}

The in vitro release procedure is described that can be used to provide assurance of product sameness between approved prechange and postchange topical dosage forms such as creams, gels, lotions and ointments.

\section{Acknowledgment}

The authors would like to appreciate and thank:

- Professor Gordon Flynn of University of Michigan and Professor Joel Zatz of Rutgers University for their scientific input in the discussions related to in vitro release.

- Dr. Robert Dillard of GD Searle and Mr. Dave Pearce of Altana for their input and discussions in statistical evaluation of comparative in vitro release data.

- Dr. Avi Yacobi Taro Pharmaceuticals, Dr. Michael Corbo of RW Johnson, Dr. Prakash Parab of Bristol Meyers Squibb and Ms. Deborah Miran of Alpharma for their input in the in vitro release discussions.

\section{Referentes}

1. Guidance for industry: S U P A C - S S nonsterile semisolid dosage forms. Scale-up and postapproval changes: chemistry, manufacturing, and controls; in vitro release testing and in vivo bioequivalence documentation. US Department of Health and Human Services, Food and Drug Administration, Center for Drug Evaluation and Research, May 1997.

2.Workshop on: Assessment of Value and Applications of in vitro Testing of Topical Dermatological Drug Products, sponsored by American Association of Pharmaceutical Scientists and FDA: September 1997.

3. Workshop report: Scale-Up of Liquid and Semisolid Disperse Systems. Van Buskirk, G.A., V. P. Shah, D. Adair et.al. Pharmaceutical Research, 11, 12161220, 1994.

4. Shah, V. P., J. Elkins, J. Hanus, C. Noorizadeh, and J. P. Skelly,"In Vitro Release of Hydrocortisone from Topical Preparations and Automated Procedure," Pharmaceutical Research, 8:55-59, 1991.

5.Shah, V. P., J. S.

Elkins, and R. L.

Williams, "In Vitro Drug Release Measurement of Topical Glucocorticoid Creams," Pharmacopeial Forum, 19, 5048-5059, 1993.

6. Corbo, M., T. W. Schultz, G. K. Wong, and G. A. Van Buskirk, "Development and Validation of In Vitro Release Testing Methods for Semisolid Formulations," Pharmaceutical Technology 17(9):112-128, 1993.

7. Li, J. B. and P. C. Rahn, "Automated Dissolution Testing of Topical Drug Formulations Using Franz Cells and HPLC Analysis," Pharmaceutical Technology 17(7):44-52, 1993.

8. Shah, V. P. and J. S. Elkins, "In Vitro Release from Corticosteroid Ointments," Fournal of Pharmaceutical Sciences, 84:11391140, 1995.
9. Zatz, J.L., "Drug Release from Semisolids: Effect of Membrane Permeability on Sensitivity to Product Parameters," Pharmaceutical Research 2:787-789, 1995.

10. Zatz, J.L., and J. D. Segers, "Techniques for measuring in vitro release from semisolids." Dissolution Technologies, 5 (1, February), 3-17, 1998.

11. Conover, W.J., Practical Nonparametric Statistics (Second Edition), John Wiley \& Sons, page $223 \mathrm{ff}, 1980$.

12. Hollander, M. and D.A.Wolfe, Nonparametric Statistical Methods, John Wiley \& Sons, page 78ff, 1973. 